\title{
Investigation of the internal structure and dynamics of gum cancer cells, tissues and tumors by 13C-NMR spectra of DNA/RNA of gum cancer cells as an essential structural tool for integrative studies of gum cancer cells development
}

\author{
Alireza Heidari ${ }^{1,2 *}$ and Ricardo Gobato ${ }^{3}$ \\ ${ }^{1}$ Faculty of Chemistry, California South University, 14731 Comet St. Irvine, CA 92604, USA \\ ${ }^{2}$ American International Standards Institute, Irvine, CA 3800, USA \\ ${ }^{3}$ Green Land Landscaping and Gardening, Seedling Growth Laboratory, 86130-000, Parana, Brazil
}

\begin{abstract}
In the current paper, investigation of the internal structure and dynamics of gum cancer cells, tissues and tumors by 13C-NMR spectra of DNA/RNA of gum cancer cells as an essential structural tool for integrative studies of gum cancer cells development are studied. Internal structure and dynamics of gum cancer cells, tissues and tumors by 13C-NMR spectra of DNA/RNA of gum cancer cells as an essential structural tool for integrative studies of gum cancer cells development-based diagnostics and imaging offer many advantages over the routine clinical techniques non-invasiveness, tissue removing is not required, minimal sample processing, labeling is not required, no prior knowledge to target molecules (e.g. antibodies) is required, safety, non-ionizing electromagnetic field is used, no external dyes/ contrast agents are needed to produce human organs images. The method is faster and cheaper to perform, which will result in quicker patient diagnosis, fewer time delays, less pain, and trauma of patients, less cost to the hospital. Investigation of the internal structure and dynamics of gum cancer cells, tissues and tumors by 13C-NMR spectra of DNA/RNA of gum cancer cells as an essential structural tool for integrative studies of gum cancer cells development based methods with the sensitivity and specificity of over $90 \%$ for in vivo and ex vivo measurements might be applied to clinical practice with a positive economic impact due to reducing of false biopsy and risk of incomplete tumor resection.
\end{abstract}

\section{Introduction}

It has been showed that internal structure and dynamics of gum cancer cells, tissues and tumors by 13C-NMR spectra of DNA/RNA of gum cancer cells as an essential structural tool for integrative studies of gum cancer cells development measurements of the biochemical mapping of human gum cancer tissues and cell cultures reveals unique internal structure and dynamics of gum cancer cells, tissues and tumors by $13 \mathrm{C}-\mathrm{NMR}$ spectra of DNA/RNA of gum cancer cells as an essential structural tool for integrative studies of gum cancer cells development fingerprints that discriminate normal and gum cancer cells, monitor epigenetic modifications-acetylation and methylation processes in gum cancer cells and tissues, gum cancer phenotype, angiogenesis in gum cancer, lipid reprogramming, response to drug treatment and glycome profile. Internal structure and dynamics of gum cancer cells, tissues and tumors by 13C-NMR spectra of DNA/RNA of gum cancer cells as an essential structural tool for integrative studies of gum cancer cells development imaging combined with AFM shows that ontogenically transformed cells demonstrate significant specific alterations in chemical composition and nanomechanical-stiffness and adhesion features. Internal structure and dynamics of gum cancer cells, tissues and tumors by 13C-NMR spectra of DNA/RNA of gum cancer cells as an essential structural tool for integrative studies of gum cancer cells development imaging together with internal structure and dynamics of gum cancer cells, tissues and tumors by 13C-NMR spectra of DNA/RNA of gum cancer cells as an essential structural tool for integrative studies of gum cancer cells development is revealing dynamics in normal and gum cancerous cells at a new molecular level. These multidisciplinary approaches for cell cultures, ex vivo tissues, animal models can be helpful in translations of all scientific findings to oncological clinical practice [1-10].

\section{Results and discussion}

Currently, internal structure and dynamics of gum cancer cells, tissues and tumors by 13C-NMR spectra of DNA/RNA of gum cancer cells as an essential structural tool for integrative studies of gum cancer cells development of linear and non-linear optical methods for gum cancer detection provides a valuable tool to improve sensitivity and specificity. One of the main reasons for insufficient progress in gum cancer diagnostics is related to the fact that most gum cancer types are not only heterogeneous in their genetic composition but also reside

${ }^{*}$ Correspondence to: Alireza Heidari, Faculty of Chemistry, California South University, 14731 Comet St. Irvine, CA 92604; American International Standards Institute, Irvine, CA 3800, USA, E-mail: Scholar.Researcher.Scientist@gmail. com; Alireza.Heidari@calsu.us; Central@aisi-usa.org

Key words: internal structure, dynamics, gum cancer cells, gum cancer tissues, tumors, 13C-NMR spectra, DNA/RNA, gum cancer cells

Received: September 16, 2020; Accepted: October 02, 2020, Published: October 05,2020 

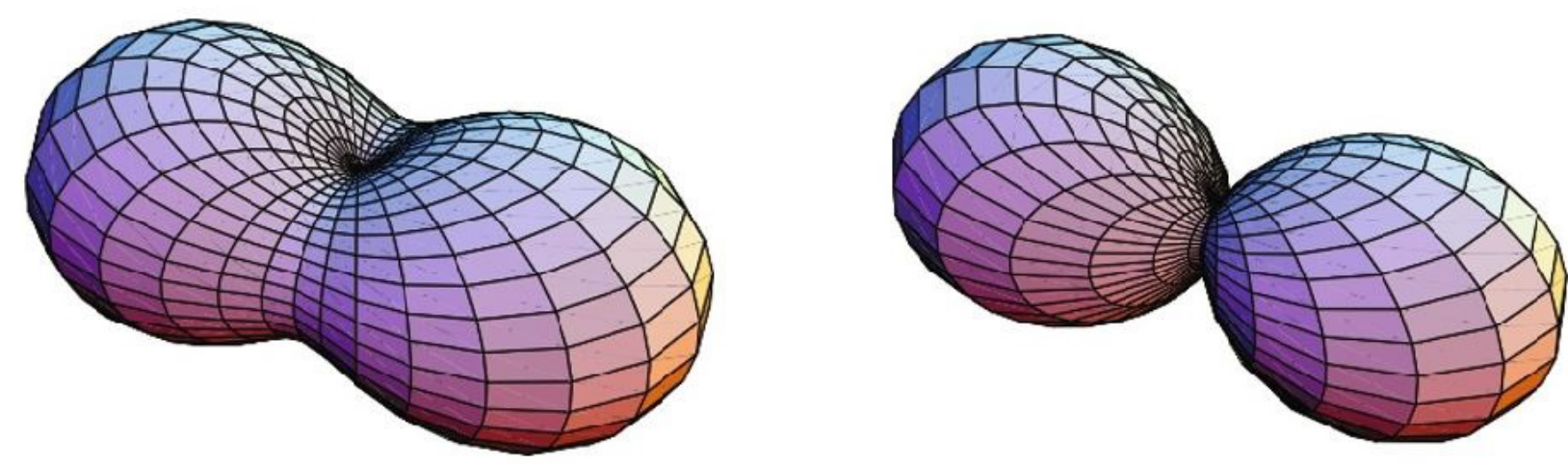

Figure 1. Internal structure and dynamics of gum cancer cells, tissues and tumors by 13C-NMR spectra of DNA/RNA of gum cancer cells

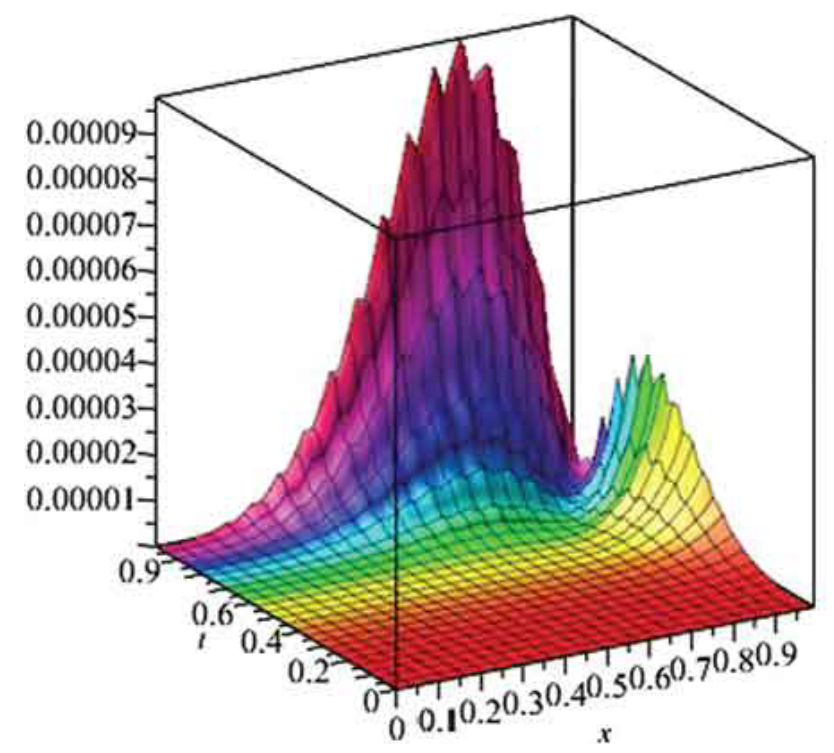

Figure 2. External structure and dynamics of gum cancer cells, tissues and tumors by 13C-NMR spectra of DNA/RNA of gum cancer cells

in varying microenvironments and interact with different cell types. Until now, no technology has been fully proven for effective detecting of invasive gum cancer, which infiltrating the extracellular matrix (Figures 1 and 2).

\section{Conclusion}

This review investigates the current status of internal structure and dynamics of gum cancer cells, tissues and tumors by 13C-NMR spectra of DNA/RNA of gum cancer cells as an essential structural tool for integrative studies of gum cancer cells development and internal structure and dynamics of gum cancer cells, tissues and tumors by $13 \mathrm{C}-$ NMR spectra of DNA/RNA of gum cancer cells as an essential structural tool for integrative studies of gum cancer cells development for gum cancer diagnostics. Moreover, the review provides a comprehensive overview of the applicability of internal structure and dynamics of gum cancer cells, tissues and tumors by 13C-NMR spectra of DNA/RNA of gum cancer cells as an essential structural tool for integrative studies of gum cancer cells development, linear and non-linear optics in gum cancer research as a gateway to tumor cell identity.

\section{Acknowledgments}

This study was supported by the Cancer Research Institute (CRI) Project of Scientific Instrument and Equipment Development, the National Natural Science Foundation of the United Sates, the International Joint BioSpectroscopy Core Research Laboratory Program supported by the California South University (CSU), and the Key project supported by the American International Standards Institute (AISI), Irvine, California, USA.

\section{References}

1. Heidari A, Brown C (2015) Study of composition and morphology of cadmium oxide (CdO) nanoparticles for eliminating cancer cells. J Nanomed Res 2(5): 20.

2. Heidari A, Brown C (2015) Study of surface morphological, phytochemical and structural characteristics of rhodium (III) oxide $\left(\mathrm{Rh}_{2} \mathrm{O}_{3}\right)$ nanoparticles. International Journal of Pharmacology, Phytochemistry and Ethnomedicine 1(1): 15-19.

3. Heidari A (2016) An experimental biospectroscopic study on seminal plasma in determination of semen quality for evaluation of male infertility. Int J Adv Technol 7: $\mathrm{e} 007$.

4. Heidari A (2016) Extraction and preconcentration of N-Tolyl-Sulfonyl-PhosphoramidSaeure-Dichlorid as an anti-cancer drug from plants: a pharmacognosy study. $J$ Pharmacogn Nat Prod 2: e103. 
Heidari A (2020) Investigation of the internal structure and dynamics of gum cancer cells, tissues and tumors by 13C-NMR spectra of DNA/RNA of gum cancer cells as an essential structural tool for integrative studies of gum cancer cells development

5. Heidari A (2016) A thermodynamic study on hydration and dehydration of DNA and RNA-Amphiphile Complexes. J Bioeng Biomed Sci S: 006.

6. Heidari A (2016) Computational studies on molecular structures and carbonyl and ketene groups' effects of singlet and triplet energies of Azidoketene $\mathrm{O}=\mathrm{C}=\mathrm{CH}-\mathrm{NNN}$ and Isocyanatoketene $\mathrm{O}=\mathrm{C}=\mathrm{CH}-\mathrm{N}=\mathrm{C}=\mathrm{O}$. $J$ Appl Computat Math 5: e142.

7. Heidari A (2016) Study of irradiations to enhance the induces the dissociation of hydrogen bonds between peptide chains and transition from helix structure to random coil structure using ATR-FTIR, Raman and ${ }^{~}$ HNMR Spectroscopies. J Biomol Res Ther 5: e146.
8. Heidari A (2016) Future prospects of point fluorescence spectroscopy, fluorescence imaging and fluorescence endoscopy in photodynamic therapy (PDT) for cancer cells. J Bioanal Biomed 8: e135.

9. Heidari A (2016) A bio-spectroscopic study of DNA density and color role as determining factor for absorbed irradiation in cancer cells. Adv Cancer Prev 1: e102.

10. Heidari A (2016) Manufacturing process of solar cells using cadmium oxide (CdO) and rhodium (III) Oxide $\left(\mathrm{Rh}_{2} \mathrm{O}_{3}\right)$ nanoparticles. J Biotechnol Biomater 6: e125.

Copyright: $\odot 2020$ Heidari A. This is an open-access article distributed under the terms of the Creative Commons Attribution License, which permits unrestricted use, distribution, and reproduction in any medium, provided the original author and source are credited. 